\title{
Decision tree analysis in subarachnoid hemorrhage: prediction of outcome parameters during the course of aneurysmal subarachnoid hemorrhage using decision tree analysis
}

\author{
Isabel Charlotte Hostettler, MD,, Carl Muroi, MD, ${ }^{3}$ Johannes Konstantin Richter, MD, ${ }^{4,5}$ \\ Josef Schmid, MA, ${ }^{6}$ Marian Christoph Neidert, MD, ${ }^{1}$ Martin Seule, MD, ${ }^{3,7}$ Oliver Boss, MD, ${ }^{3}$ \\ Athina Pangalu, MD, ${ }^{4}$ Menno Robbert Germans, MD, PhD, ${ }^{1}$ and Emanuela Keller, MD ${ }^{1,3}$ \\ Departments of ${ }^{1}$ Neurosurgery and ${ }^{4}$ Neuroradiology and ${ }^{3}$ Neurocritical Care Unit, Department of Neurosurgery, University \\ Hospital Zurich; ${ }^{5}$ Department of Diagnostic, Interventional and Pediatric Radiology, University Hospital of Bern; ${ }^{6}$ Dynelytics, \\ Zurich; 'Department of Neurosurgery, Kantonsspital St. Gallen, Switzerland; and ${ }^{2}$ Stroke Research Centre, University College \\ London, Institute of Neurology, London, United Kingdom
}

OBJECTIVE The aim of this study was to create prediction models for outcome parameters by decision tree analysis based on clinical and laboratory data in patients with aneurysmal subarachnoid hemorrhage (aSAH).

METHODS The database consisted of clinical and laboratory parameters of 548 patients with aSAH who were admitted to the Neurocritical Care Unit, University Hospital Zurich. To examine the model performance, the cohort was randomly divided into a derivation cohort ( $60 \%$ [ $n=329$ ]; training data set) and a validation cohort $(40 \%$ [ $n=219]$; test data set). The classification and regression tree prediction algorithm was applied to predict death, functional outcome, and ventriculoperitoneal (VP) shunt dependency. Chi-square automatic interaction detection was applied to predict delayed cerebral infarction on days 1,3 , and 7.

RESULTS The overall mortality was $18.4 \%$. The accuracy of the decision tree models was good for survival on day 1 and favorable functional outcome at all time points, with a difference between the training and test data sets of $<5 \%$. Prediction accuracy for survival on day 1 was $75.2 \%$. The most important differentiating factor was the interleukin-6 (IL6 ) level on day 1. Favorable functional outcome, defined as Glasgow Outcome Scale scores of 4 and 5 , was observed in $68.6 \%$ of patients. Favorable functional outcome at all time points had a prediction accuracy of $71.1 \%$ in the training data set, with procalcitonin on day 1 being the most important differentiating factor at all time points. A total of 148 patients (27\%) developed VP shunt dependency. The most important differentiating factor was hyperglycemia on admission.

CONCLUSIONS The multiple variable analysis capability of decision trees enables exploration of dependent variables in the context of multiple changing influences over the course of an illness. The decision tree currently generated increases awareness of the early systemic stress response, which is seemingly pertinent for prognostication.

https://thejns.org/doi/abs/10.3171/2017.7.JNS17677

KEY WORDS subarachnoid hemorrhage; decision tree analysis; clinical outcome; death; delayed cerebral infarction; shunt dependency; vascular disorders

$\mathrm{C}$ LINICAL outcome in aneurysmal subarachnoid hemorrhage (aSAH) is significantly influenced by complications such as rebleeding, cerebral vasospasm, delayed cerebral ischemia (DCI), secondary infarction, and hydrocephalus. ${ }^{3}$ To predict complications and clinical outcome after aSAH, several clinical grading systems, e.g., Hunt and Hess: ${ }^{10}$ World Federation of Neurosurgical Societies (WFNS); ${ }^{25}$ HAIR (Hunt and Hess, age, intraventricu- lar hemorrhage, rebleed); ${ }^{14}$ and FRESH (Functional Recovery Expected after Subarachnoid Hemorrhage); ${ }^{28}$ and radiological scores to predict cerebral vasospasm, such as Fisher grade ${ }^{5}$ and Barrow Neurological Institute (BNI) score, ${ }^{27}$ have been developed. However, their predictive accuracy remains limited. The complex pathogenesis and pleomorphic nature of the aforementioned complications certainly contribute to this fact. Nevertheless, the possibil-

ABBREVIATIONS aSAH = aneurysmal subarachnoid hemorrhage; $\mathrm{BNI}=$ Barrow Neurological Institute; $\mathrm{CRP}=\mathrm{C}$-reactive protein; $\mathrm{DCl}=$ delayed cerebral ischemia; $\mathrm{GOS}=$ Glasgow Outcome Scale; IL-6 = interleukin-6; PCT = procalcitonin; VP = ventriculoperitoneal; WFNS = World Federation of Neurosurgical Societies.

SUBMITTED March 16, 2017. ACCEPTED July 6, 2017.

INCLUDE WHEN CITING Published online January 19, 2018; DOI: 10.3171/2017.7.JNS17677. 
ity of including prediction algorithms for specific complications in daily clinical assessments would allow crucial proactive decision making in the individual patient.

The multivariable analysis capability of decision trees makes it possible to go beyond simple cause and effect relationships and to explore dependent variables in the context of multiple influences over time. ${ }^{19,22}$ The aim of this study was to create prediction models for outcome parameters using decision tree analysis based on easily accessible clinical and, in particular, laboratory data. In a decision tree analysis, patients are split in a recursive manner based on the values of a statistically important variable. The first splitting of the entire patient population into subgroups is based on the most important variable. The splitting is repeated, and the subsequent subgroups are further divided on its most important variable until the subgroup is no longer subject to further splitting.

\section{Methods \\ Database}

The database consisted of prospectively collected data from 548 patients with confirmed aSAH who were admitted to the Neurocritical Care Unit, University Hospital Zurich (an academic tertiary care center) from January 2007 to December 2013. In brief, the diagnosis was based on CT and catheter or CT angiography findings. In addition to patient's demographic characteristics, comorbidities were assessed (Table 1). The clinical severity at the time of admission was assessed according to the WFNS and Hunt and Hess grading systems. ${ }^{10,25} \mathrm{~A}$ neuroradiologist assessed the CT scans to determine the blood load (diameter of blood in a vertical cistern), Fisher grade, and BNI score, as well as the presence of infarcts. Delayed cerebral infarction was defined as radiologically or otherwise proven new infarcts, which did not occur within 48 hours of aneurysm coiling or clipping. ${ }^{4}$ We explicitly decided to define delayed cerebral infarction as an outcome variable as opposed to the more commonly used delayed cerebral ischemia (DCI), as the definition and use of this abbreviation has been inconsistent. ${ }^{26}$

The database included laboratory data, from which the following parameters were selected for the decision tree analysis: glucose level at the time of admission, and interleukin-6 (IL-6), procalcitonin (PCT), C-reactive protein (CRP) levels, and leukocyte counts on days 1,3 , and 7 after hemorrhage. Glucose level on admission was selected because it has previously been described as a predictor for poor outcome after SAH. ${ }^{13,21}$ Hyperglycemia on admission was defined as a blood glucose level greater than $8 \mathrm{mmol} / \mathrm{L}$. IL-6, PCT, CRP levels, and leukocyte counts were collected, as these systemic inflammatory parameters have been reported to correlate with occurrence of ischemic complications and/or poor clinical outcome. ${ }^{9,12,15,16,18}$ Patients underwent routine follow-up in the neurosurgical outpatient clinics 1 year after aSAH. The functional outcome was assessed according to the Glasgow Outcome Scale (GOS). ${ }^{11}$ The registry was approved by the ethics committee of $\mathrm{Zu}$ rich, Switzerland.

\section{Statistical Analysis}

Continuous variables are presented as mean \pm standard deviation and categorical variables as frequency and percent- ages. Statistical analysis was performed using IBM SPSS and IBM SPSS Modeler (versions 24 and 18, respectively).

\section{Selection of Predictors}

Univariable analysis was performed to screen candidate predictors regarding prediction of the following dependent variables: death, GOS score, occurrence of delayed cerebral infarction, and ventriculoperitoneal (VP) shunt dependency. All aforementioned clinical, radiological, and laboratory data were evaluated (Table 1). GOS scores were dichotomized as 1-3 (unfavorable outcome) and 4 and 5 (favorable outcome) for analysis. The importance of each variable was defined as $(1-p)$, with $p$ being the $p$ value of the appropriate statistical test of association between the candidate predictor and the target variable. The Pearson's chi-square test was used for categorical predictors, and the continuous 1-way ANOVA F-test was used for continuous variables. To identify the most important predictors to be included in the model, the respective $p$ values were subsequently ranked in ascending order.

\section{Decision Tree Development and Internal Validation}

To examine the model performance, the cohort was randomly divided into a derivation cohort $(60 \%$ [ $n=329]$, training data set) and a validation cohort $(40 \%[\mathrm{n}=219]$, test data set). The classification and regression tree prediction algorithm was applied to predict death, dichotomized functional outcome, and VP shunt dependency. Chi-square automatic interaction detection was applied to predict delayed cerebral infarction. ${ }^{2}$ The prediction model for each of the outcome parameters was adapted for the specific time points of days 1,3 , and 7 after aSAH.

\section{Missing Values}

If the dependent variable of a case was missing, the case was ignored in the analysis. If all predictor variables of a case were missing, the case was ignored. With the classification and regression tree algorithm, the surrogate split method was otherwise used to deal with missing data in predictor variables. With the chi-square automatic interaction detection algorithm, missing values were treated as a predictor category. Based on the smallest $\mathrm{p}$ value, the algorithm decided whether to merge the missing category with its most similar category or to keep the missing category as a separate category. ${ }^{2}$

\section{Model Presentation}

For each of the dependent variables, a risk chart was created with regard to the absence or presence of the independent variables. The group containing all patients is termed "root," and the subgroups are termed "nodes." Starting at the root, an appropriate primary split was selected, after which the data set was further divided into smaller subsets at each node up to the point where no further information could be gained or the stopping criterion was reached. The most significant independent variable tested is indicated below the root or node. In the branches to the next node, corresponding threshold values of this independent variable can be found. The improvement of the model with each additional layer can be estimated by the numerical value below the label of the independent variable. The performance of each model was assessed by comparing its 
TABLE 1. Patients' clinical and radiological characteristics (independent variables)

\begin{tabular}{|c|c|}
\hline Variable & Value \\
\hline \multicolumn{2}{|l|}{ Sex } \\
\hline Female & $368(67.2)$ \\
\hline Male & $226(32.8)$ \\
\hline Age, mean \pm SD in yrs & $54.7 \pm 13.3$ \\
\hline \multicolumn{2}{|l|}{ Comorbidities, $n(\%)$} \\
\hline Arterial hypertension & $193(35.2)$ \\
\hline Hypercholesterolemia & $52(9.5)$ \\
\hline Diabetes mellitus & $21(3.8)$ \\
\hline Heart disorder & $46(8.4)$ \\
\hline Neurological disorder & $32(5.8)$ \\
\hline Liver disorder & $61(11.1)$ \\
\hline Migraine & $34(6.2)$ \\
\hline Smoking & $252(46.0)$ \\
\hline Recreational drug use & $20(3.7)$ \\
\hline Malignancy & $4(0.7)$ \\
\hline \multicolumn{2}{|l|}{ Prior medication, $\mathrm{n}(\%)$} \\
\hline Anticoagulation & $17(3.1)$ \\
\hline Antiplatelet drugs & 79 (14.4) \\
\hline \multicolumn{2}{|l|}{ Clinical baseline characteristics } \\
\hline \multicolumn{2}{|l|}{ WFNS grade, $\mathrm{n}(\%)$} \\
\hline I & $144(26.1)$ \\
\hline II & $178(32.5)$ \\
\hline III & $81(14.8)$ \\
\hline IV & $77(14.1)$ \\
\hline V & $68(12.4)$ \\
\hline \multicolumn{2}{|l|}{ Radiological baseline characteristics } \\
\hline \multicolumn{2}{|l|}{ Fisher grade, $\mathrm{n}(\%)$} \\
\hline 1 & $13(2.6)$ \\
\hline 2 & $9(1.8)$ \\
\hline 3 & $378(74.6)$ \\
\hline 4 & $107(21.1)$ \\
\hline \multicolumn{2}{|l|}{ BNI score, $n(\%)$} \\
\hline 1 & $13(2.6)$ \\
\hline 2 & $42(8.3)$ \\
\hline 3 & $123(24.3)$ \\
\hline 4 & $227(44.8)$ \\
\hline 5 & $102(20.1)$ \\
\hline Blood diameter in vertical cistern, mean $\pm \mathrm{SD}$ in $\mathrm{mm}$ & $11.6 \pm 6.0$ \\
\hline \multicolumn{2}{|l|}{ Ruptured aneurysm location, $\mathrm{n}(\%)$} \\
\hline Internal carotid artery & $64(11.7)$ \\
\hline Middle cerebral artery & $124(22.6)$ \\
\hline Anterior cerebral artery & $4(0.7)$ \\
\hline Anterior communicating artery & $178(32.5)$ \\
\hline Pericallosal artery & $24(4.4)$ \\
\hline Posterior cerebral artery & $9(1.6)$ \\
\hline Posterior communicating artery & $75(13.7)$ \\
\hline Posterior inferior cerebellar artery & $19(3.5)$ \\
\hline Anterior inferior cerebellar artery & $2(0.4)$ \\
\hline Basilar artery & $32(5.8)$ \\
\hline
\end{tabular}

» CONTINUED FROM PREVIOUS COLUMN

TABLE 1. Patients' clinical and radiological characteristics (independent variables)

\begin{tabular}{lr}
\hline \multicolumn{1}{c}{ Variable } & Value \\
\hline Ruptured aneurysm location, $\mathrm{n}(\%)$ (continued) & \\
\hline Vertebral artery & $13(2.4)$ \\
\hline Anterior choroidal artery & $3(0.5)$ \\
\hline$\quad$ Superior cerebellar artery & $1(0.2)$ \\
\hline Multiple aneurysms, $\mathrm{n}(\%)$ & $132(24.1)$ \\
\hline Aneurysm treatment modality, $\mathrm{n}(\%)$ & \\
\hline Aneurysm clipping & $288(52.5)$ \\
\hline Aneurysm coiling & $214(39.0)$ \\
\hline Combination of clipping \& coiling & $13(2.4)$ \\
\hline Conservative & $31(5.7)$ \\
\hline Bypass \& trapping & $2(0.4)$ \\
\hline Occurrence of rebleeding & $43(7.8)$ \\
\hline
\end{tabular}

sensitivity and specificity in predicting a specific event in both the training and test data sets. The quality of prediction was estimated to be good if the difference between the training and test data sets was $<5 \%$ and acceptable if it was $<10 \%$. Based on the recorded factors of a new patient, risk can be predicted by following the decision tree path, beginning from the root to the nodes.

\section{Results}

The mean age of all patients was $54.7 \pm 13.25$ years (range 14-87 years); 368 patients $(67.2 \%)$ were female and $180(32.8 \%)$ male. Of the population, $226(41.2 \%)$ patients sustained a high-grade SAH (WFNS grades III-V), and $320(58.4 \%)$ a low-grade SAH (WFNS grades I and II). Two hundred eighty-eight (52.5\%) patients were treated with clipping, $214(39.0 \%)$ with coiling, 13 patients had a combination of coiling and clipping (2.4\%), 2 patients received a bypass for trapping of the aneurysm $(0.4 \%)$, and 31 patients $(5.7 \%)$ were treated conservatively. Patients' baseline characteristics are shown in Table 1.

\section{Prediction of Outcome \\ Prediction of Death}

A total of 101 of 548 patients died, resulting in an overall mortality of $18.4 \%$ (Table 2). The decision tree models predicting death or survival on days 1,3 , and 7 after aSAH are shown in Figs. 1-3. The most important differentiating factor on day 1 was the IL-6 level. The pathway with

TABLE 2. Outcome characteristics (dependent variables)

\begin{tabular}{lc}
\hline \multicolumn{1}{c}{ Characteristic } & No. of Patients (\%) \\
\hline Overall mortality & $101(18.4)$ \\
\hline Outcome after 1 yr & $137(31.4)$ \\
\hline GOS scores 1-3 (unfavorable) & $300(68.6)$ \\
\hline GOS scores 4 \& 5 (favorable) & $127(23.2)$ \\
\hline Delayed infarction & $148(27.0)$ \\
\hline VP shunt dependency & \\
\hline
\end{tabular}




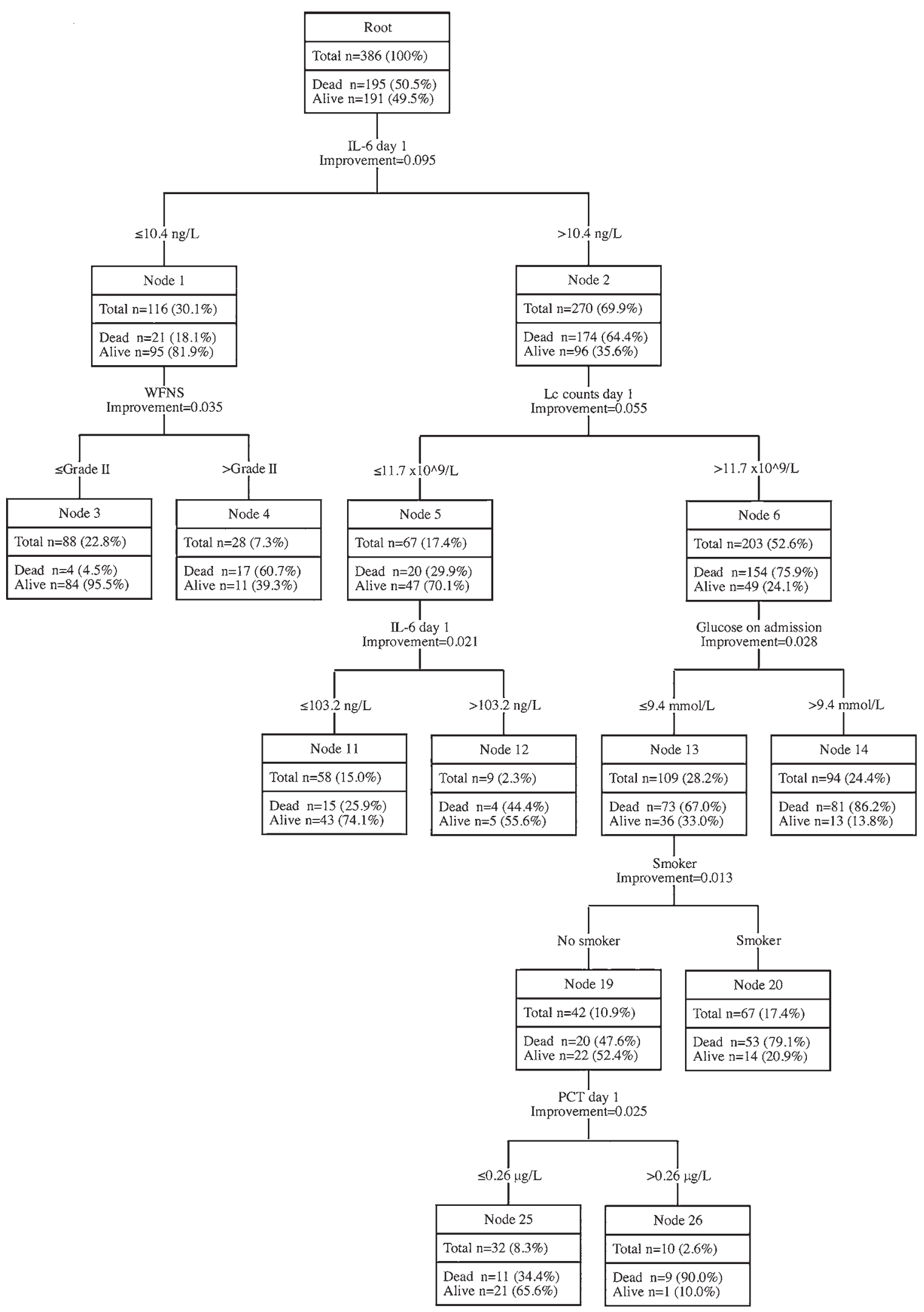

FIG. 1. Decision tree models on day 1 for the occurrence of death. LC = leukocytes. 


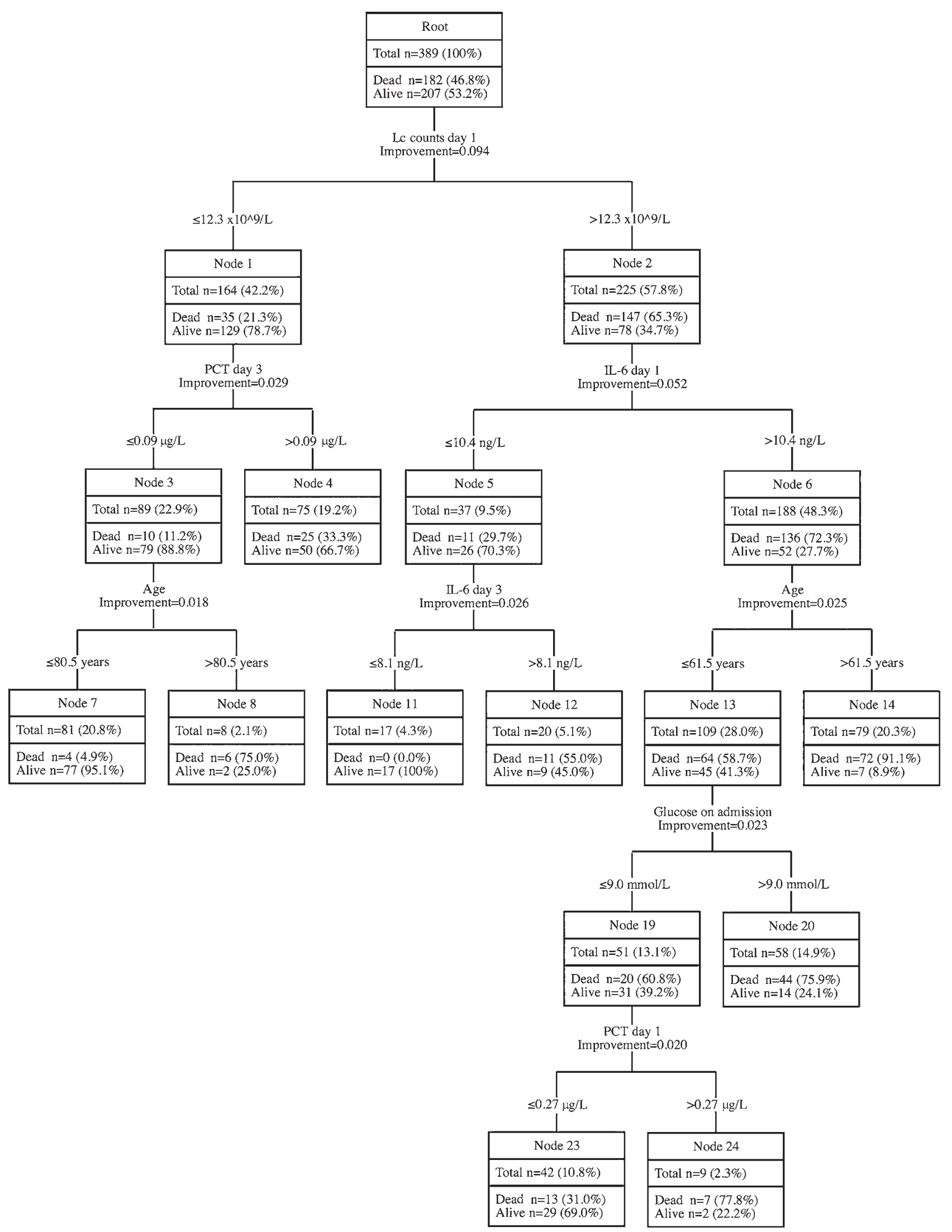

FIG. 2. Decision tree models on day 3 for the occurrence of death. 


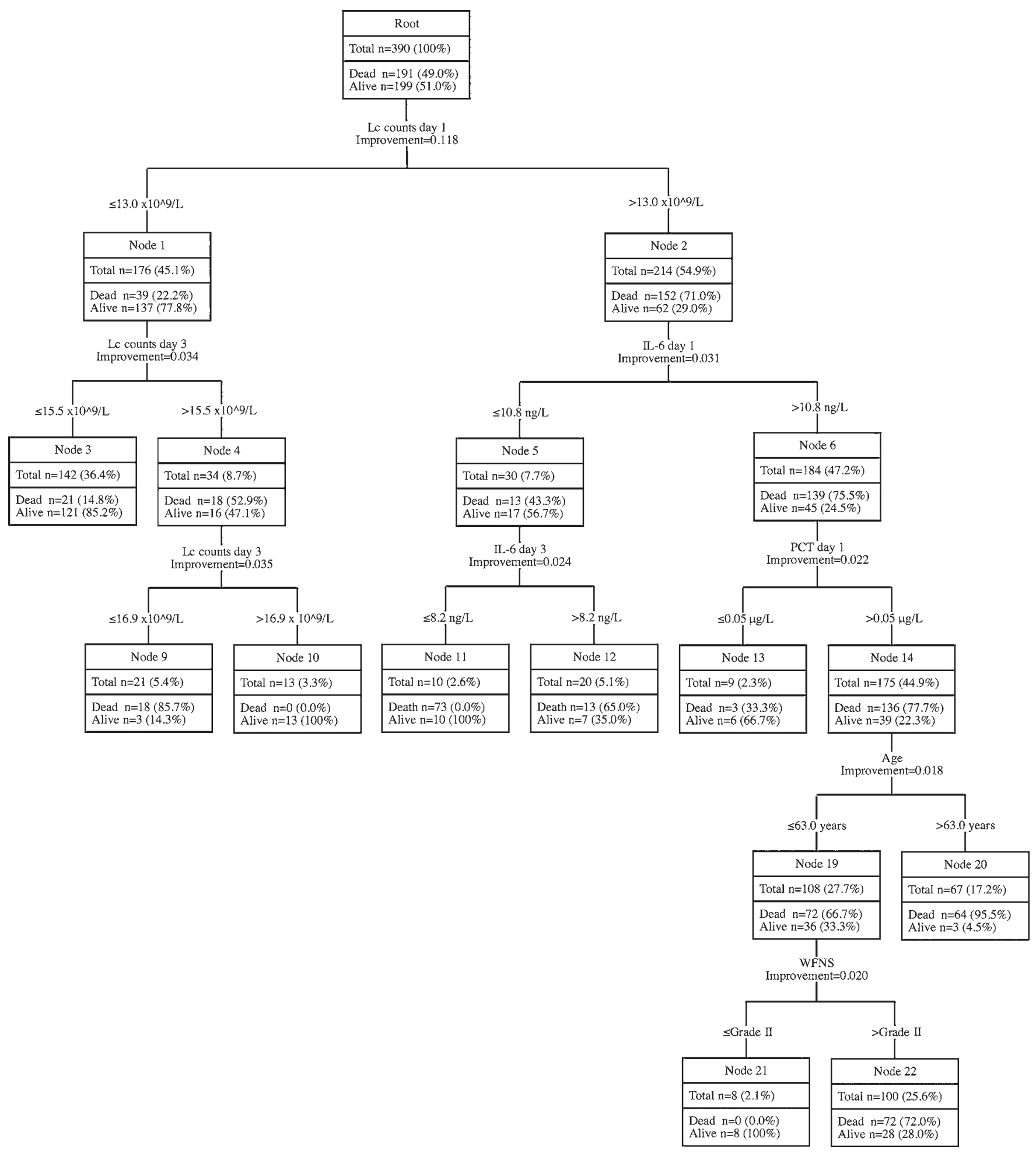

FIG. 3. Decision tree models on day 7 for the occurrence of death.

the best prediction of death was as follows: IL- 6 on day 1 greater than $10.35 \mathrm{ng} / \mathrm{L}$ (node 2), leukocyte counts on day 1 greater than $11.7 \times 10^{9} / \mathrm{L}$ (node 6 ), and glucose on admission greater than $9.4 \mathrm{mmol} / \mathrm{L}$ (node 14). The model had the greatest accuracy on day 1 . The sensitivity to predict death was $83.1 \%$ in the training and $60 \%$ in the test data set, and the specificity was $75.3 \%$ and $71.0 \%$, respectively (Table 3 ). The most important differentiating factor on days 3 and 7 was the leukocyte count on day 1 . The specificity was $83.0 \%$ in the training and $76.7 \%$ in the test data set on day 3 , and $78.2 \%$ in the training and $71.6 \%$ in the test set on day 7 . 
TABLE 3. Performance of the models for death, dichotomized functional outcome, delayed cerebral infarction, and VP shunt dependency on days 1,3 , and 7

\begin{tabular}{|c|c|c|}
\hline Outcome Parameter & $\begin{array}{l}\text { Training } \\
\text { Data Set }\end{array}$ & $\begin{array}{l}\text { Test Data } \\
\text { Set }\end{array}$ \\
\hline \multicolumn{3}{|l|}{ Death } \\
\hline \multicolumn{3}{|l|}{ Model on day 1} \\
\hline $\begin{array}{l}\text { Correct prediction of death, } \mathrm{n} \\
\text { (sensitivity) }\end{array}$ & $51(83.1 \%)$ & $24(60.0 \%)$ \\
\hline $\begin{array}{l}\text { Correct prediction of survival, } \mathrm{n} \\
\text { (specificity) }\end{array}$ & $204(75.3 \%)$ & $125(71.0 \%)$ \\
\hline \multicolumn{3}{|l|}{ Model on day 3} \\
\hline $\begin{array}{l}\text { Correct prediction of death, } n \\
\text { (sensitivity) }\end{array}$ & $46(75.4 \%)$ & $20(50.0 \%)$ \\
\hline $\begin{array}{l}\text { Correct prediction of survival, } \mathrm{n} \\
\text { (specificity) }\end{array}$ & $225(83.0 \%)$ & $135(76.7 \%)$ \\
\hline \multicolumn{3}{|l|}{ Model on day 7} \\
\hline $\begin{array}{l}\text { Correct prediction of death, } \mathrm{n} \\
\text { (sensitivity) }\end{array}$ & $52(85.3 \%)$ & $22(55.0 \%)$ \\
\hline $\begin{array}{l}\text { Correct prediction of survival, } \mathrm{n} \\
\text { (specificity) }\end{array}$ & $212(78.2 \%)$ & $126(71.6 \%)$ \\
\hline \multicolumn{3}{|l|}{ Dichotomized functional outcome } \\
\hline \multicolumn{3}{|l|}{ Model on days $1,3, \& 7$} \\
\hline $\begin{array}{l}\text { Correct prediction of favorable } \\
\text { outcome, } \mathrm{n} \text { (sensitivity) }\end{array}$ & $128(71.1 \%)$ & $80(66.7 \%)$ \\
\hline $\begin{array}{l}\text { Correct prediction of unfavorable } \\
\text { outcome, } n \text { (sensitivity) }\end{array}$ & $63(82.9 \%)$ & $46(75.4 \%)$ \\
\hline \multicolumn{3}{|l|}{ Delayed cerebral infarction } \\
\hline \multicolumn{3}{|l|}{ Model on days $1,3, \& 7$} \\
\hline $\begin{array}{l}\text { Correct prediction of occurrence } \\
\text { of delayed cerebral infarction } \\
\text { (sensitivity) }\end{array}$ & $37(52.1 \%)$ & $25(44.6 \%)$ \\
\hline $\begin{array}{l}\text { Correct prediction of absence } \\
\text { of delayed cerebral infarction } \\
\text { (specificity) }\end{array}$ & $208(79.7 \%)$ & $111(69.4 \%)$ \\
\hline \multicolumn{3}{|l|}{ VP shunt dependency } \\
\hline \multicolumn{3}{|l|}{ Model on day 1} \\
\hline $\begin{array}{l}\text { Correct prediction of VP shunt } \\
\text { dependency (sensitivity) }\end{array}$ & $137(78.3 \%)$ & $43(62.3 \%)$ \\
\hline $\begin{array}{l}\text { Correct prediction of absence of VP } \\
\text { shunt dependency (specificity) }\end{array}$ & $159(62.8 \%)$ & $89(60.5 \%)$ \\
\hline \multicolumn{3}{|l|}{ Model on day 3} \\
\hline $\begin{array}{l}\text { Correct prediction of VP shunt } \\
\text { dependency (sensitivity) }\end{array}$ & $62(78.5 \%)$ & $43(62.3 \%)$ \\
\hline $\begin{array}{l}\text { Correct prediction of absence of VP } \\
\text { shunt dependency (specificity) }\end{array}$ & $159(62.9 \%)$ & $89(60.5 \%)$ \\
\hline \multicolumn{3}{|l|}{ Model on day 7} \\
\hline $\begin{array}{l}\text { Correct prediction of VP shunt } \\
\text { dependency (sensitivity) }\end{array}$ & $53(67.1 \%)$ & $2(30.4 \%)$ \\
\hline $\begin{array}{l}\text { Correct prediction of absence of VP } \\
\text { shunt dependency (specificity) }\end{array}$ & $22(87.3 \%)$ & $119(80.9 \%)$ \\
\hline
\end{tabular}

\section{Prediction of Functional Outcome}

The outcome data regarding GOS score after 1 year were available in 437 patients. Favorable functional outcome, defined as GOS scores 4 and 5, was observed in $68.6 \%$ of patients (Table 2). For the dichotomized outcome (GOS scores 4 and 5 as favorable outcome; GOS score $\leq$ 3 as unfavorable outcome), the decision tree demonstrated the same prognostic subgroups and ranking order at all time points (Fig. 4). The pathway with the best prediction of unfavorable outcome was PCT on day 1 of greater than $0.23 \mu \mathrm{g} / \mathrm{L}$ and of favorable outcome PCT on day 1 of 0.23 $\mu \mathrm{g} / \mathrm{L}$ or lower, as the most important differentiating factor (node 1), followed by WFNS grade II or lower (node 3 ). At each time point, the prediction accuracy for favorable outcome was $71.1 \%$ in the training and $66.7 \%$ in the test data set (Table 3), and the prediction accuracy for unfavorable outcome was $82.9 \%$ in the training and $75.4 \%$ in the testing data set.

\section{Prediction of Delayed Cerebral Infarction}

A total of 127 patients (23.2\%) developed delayed cerebral infarction. The decision tree revealed the same prognostic subgroups and ranking order at all time points (Fig. 5). A CRP level on day 1 of $23 \mathrm{mg} / \mathrm{L}$ or lower was the most important differentiating factor. The pathway with the best prediction of absent delayed cerebral infarction was CRP level on day 1 of less than $23 \mathrm{mg} / \mathrm{L}$ (node 1), CRP level on day 1 of $11 \mathrm{mg} / \mathrm{L}$ or lower (node 3 ), no present comorbidi-

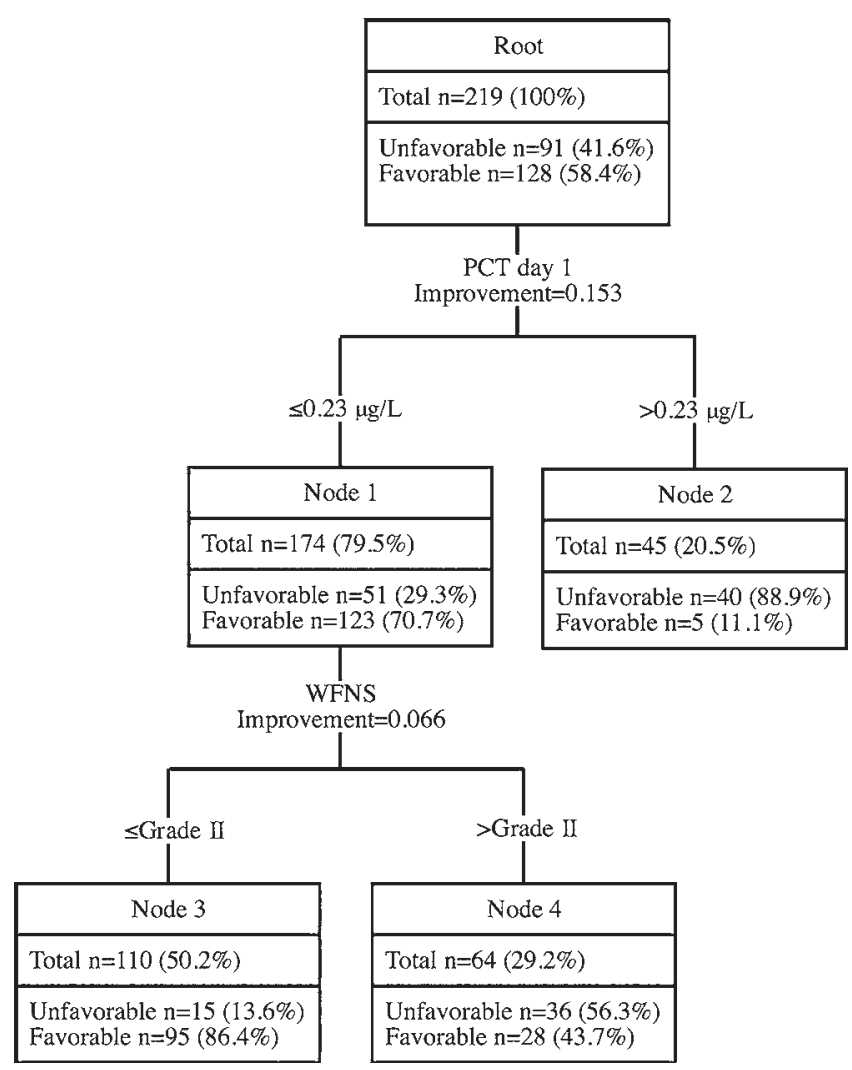

FIG. 4. Decision tree models for the dichotomized outcome were the same on days 1,3 , and 7 . GOS scores of $1-3$ were defined as "unfavorable" and GOS scores of $4-5$ as "favorable." 


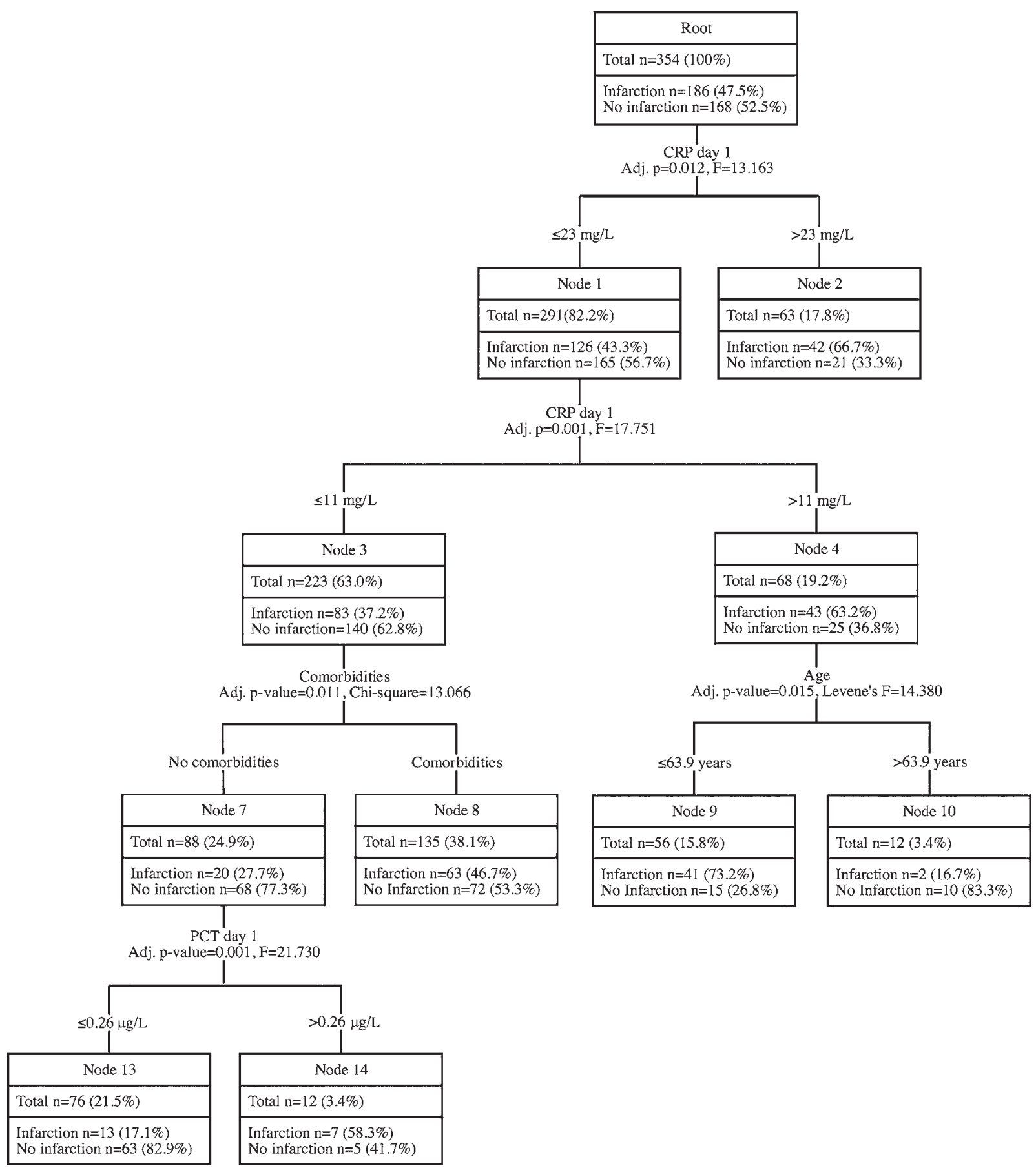

FIG. 5. Decision tree models for occurrence of delayed cerebral infarction were the same on days 1,3 , and 7 .

ties (node 7), and PCT level on day 1 of $0.26 \mu \mathrm{g} / \mathrm{L}$ or lower (node 13). The prediction accuracy for the absence of cerebral infarction was $79.7 \%$ in the training and $69.4 \%$ in the test data set at each time point (Table 3).

\section{Prediction of Shunt Dependency}

A total of 148 patients (27\%) developed VP shunt dependency. The same decision tree fit best for the first 2 time points (Fig. 6). The best prediction was achieved with the decision tree on day 7 (Fig. 7). The most important dif- ferentiating factor was hyperglycemia on admission (root), followed by the localization of the aneurysm (node 1) and WFNS grade (node 2). Overall, $89.8 \%$ of the patients with absence of hyperglycemia on admission, ruptured aneurysm in the anterior circulation, and no concomitant malignant disease did not develop VP shunt dependency. Regarding the absence of VP shunt dependency, the model was most accurate on day 7 with a prediction accuracy of $87.3 \%$ in the training set and $80.9 \%$ in the test data set (Table 3). 


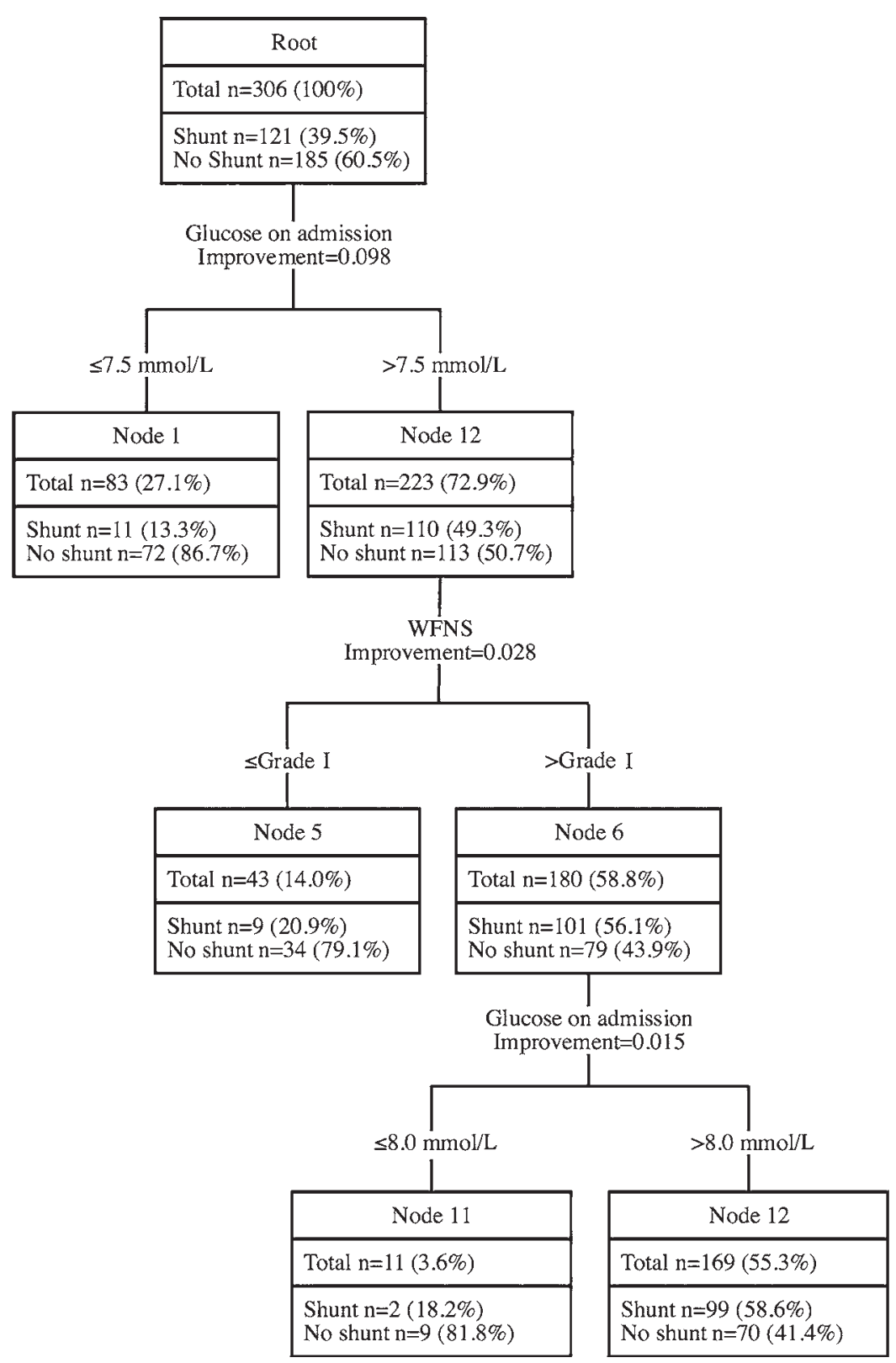

FIG. 6. Decision tree models for VP shunt dependency on days 1 and 3.

\section{Discussion}

The accuracy of the decision tree models was good for survival on day 1 and for favorable functional outcome at all 3 time points, with a difference between the training and test data set of less than 5\%. Prediction accuracy for survival on day 1 was $75.3 \%$ in the training data set. The most important differentiating factor was the IL-6 level on day 1. Systemic IL-6 levels have previously been reported to correlate with the clinical severity grade, occurrence of delayed neurological deficits, and outcome after aSAH. ${ }^{16,17}$ However, in those studies, serial measurements over the course illness were averaged and analyzed. Less is known about the predictive value of early ( $\leq 24$ hours) levels. The IL- 6 level on day 1 might reflect the severity of the initial stress response in a selective manner.

Favorable functional outcome at all time points had a prediction accuracy of $71.1 \%$ in the training data set, with PCT on day 1 being the most important differentiating factor at all time points. PCT, as an acute-phase protein, is elevated under various other acute stress conditions, such as polytrauma, cardiac arrest, and burns. ${ }^{1}$ It can be assumed that PCT levels in aSAH patients reflect an acute systemic stress response to the hemorrhage. Of further interest, early ( $\leq 24$ hours) PCT levels have been reported to accurately predict unfavorable neurological outcome after transient global cerebral ischemia due to cardiac arrest. ${ }^{6,8}$ In severe aSAH, it is known that comparable transient global cerebral ischemia occurs? 


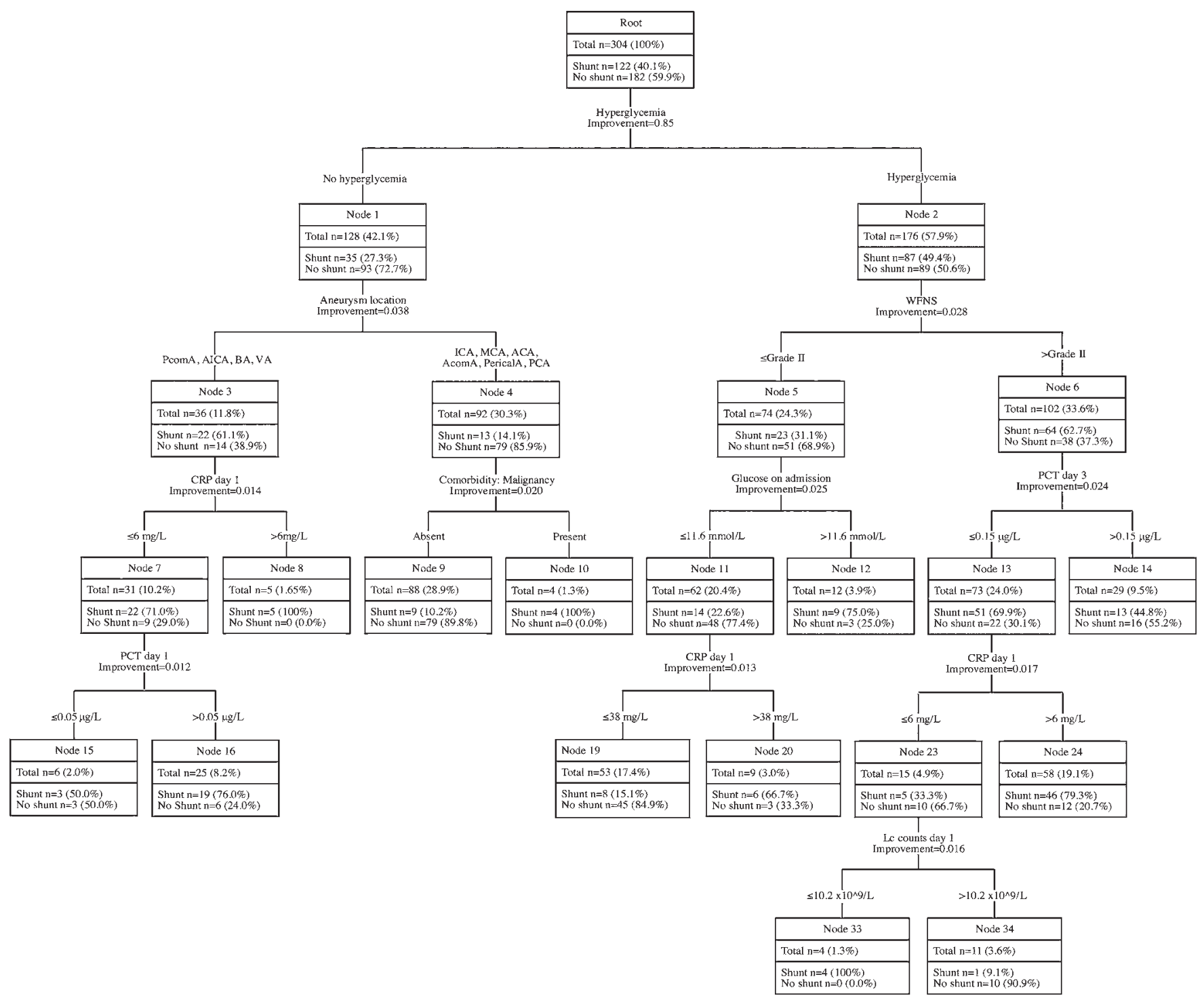

FIG. 7. Decision tree models for VP shunt dependency on day 7. ACA = anterior cerebral artery; $A$ com $A=$ anterior communicating artery; $\mathrm{AICA}=$ anterior inferior cerebellar artery; $\mathrm{BA}=$ basilar artery; $\mathrm{ICA}=$ internal carotid artery; $\mathrm{MCA}=$ middle cerebral artery; PcomA = posterior communicating artery; Perical $A=$ pericallosal artery; $V A=$ vertebral artery.

For survival, the prediction accuracy was acceptable on days 3 and 7 (difference between training and test data set $<10 \%$ ). The prediction accuracy for survival on day 3 was $83 \%$ and $78.2 \%$ on day 7 . In both cases, a high leukocyte count on day 1 was the most important differentiating factor. Leukocytosis during the course of the illness has been linked with occurrence of vasospasm, DCI, and/or outcome..$^{15,23}$ More recently, initial ( $\leq 24$ hours) leukocyte counts have been reported to be predictive for DCI and poor neurological outcome. ${ }^{24}$

The most important differentiating factor for shunt dependency was hyperglycemia on admission. This corresponds to the evaluation of the Columbia University database (published in 2010). Glucose values of at least $126 \mathrm{mg} /$ $\mathrm{dl}(7 \mathrm{mmol} / \mathrm{L})$ were found to be associated with long-term shunt dependency. ${ }^{20}$

The present study first analyzed predictors for outcome parameters in a time-dependent manner on days 1,3 , and 7. Interestingly, laboratory parameters obtained on day 1 were the most important differentiating factors in our statistical model and thereby surpassed conventional scoring systems, such as the Hunt and Hess and WFNS grading systems. This might be explained by the fact that these grades are assigned by humans and thus are subject to interrater variability, whereas laboratory parameters are absolute values. The results underline the significance of the very initial systemic stress response, reflecting the impact of the hemorrhage on the entire organism, for the overall mortality and morbidity.

In clinical practice, decision making is required on a daily and continuous basis. Even more, critical threshold values of laboratory parameters are not static but change frequently during the course of a disease, as can be seen 
in our data set regarding survival prediction on days 3 and 7. In our survival prediction model, leukocyte count on days 1,3 , and 7 was consistently the most important differentiating factor, but the threshold varied from 12.33 $\times 10^{9} / \mathrm{L}$ or lower on day 3 to $13.02 \times 10^{9} / \mathrm{L}$ or lower on day 7. Especially after aSAH, secondary inflammatory as well as reparatory processes change the requirements of the organism over the course of time. Threshold values according to which a patient is treated have to be adapted on a daily basis. Prediction scores have to take into account continuous changes in a patient's requirements depending on the patient's characteristics and current laboratory and clinical parameters, as well as complications that arise during the course of the disease, while providing an accurate prediction of the outcome variable of interest.

Our study has some limitations. Decision tree analysis is of limited use when missing data are present, which commonly occurs in medical studies. As our data set contained variables with missing values, only algorithms with well-defined criteria regarding the handling of missing values could be used. Another drawback regarding a decision tree is its difficult application on multifactorial, complicated diseases such as aSAH, as the more nodes there are, the less accurate the expected outcome is. Also, decision trees are based on expectations, which can lead to errors. A further clear limitation of the current study is the relatively low number of patients, and the fact that an external validation has not been performed. This is reflected in the restricted sensitivity for the specific event and the large differences between the training and test data sets as in the prediction of delayed cerebral infarctions. Therefore, it is of great interest to target these limitations with a harmonized large multicenter database to 1) perform an external validation and 2) increase the power and sensitivity of the analysis.

Despite the stated limitations, we have created a starting point for future large, multicenter studies to develop more sensitive and specific prognostic scores in patients with aSAH.

\section{Conclusions}

Prediction scores are powerful tools regarding the management and decision-making process in general and especially in patients with complex, multifactorial diseases, such as aSAH. The current study demonstrates the potential benefit of decision tree analysis in patients with aSAH. The necessity to handle an increasing amount of information calls for tools that can process and analyze large amounts of data, i.e., from continuously measured parameters over the course of an illness. Decision tree analysis is an interesting tool that can be used in data mining to generate new information based on an existing database, such as the one presented. The decision tree currently generated increases awareness of the early systemic stress response, which is seemingly pertinent for prognostication. To increase its power, sensitivity, and specificity, its accuracy needs to be validated in future studies of large cohorts.

\section{References}

1. Becker KL, Snider R, Nylen ES: Procalcitonin assay in sys- temic inflammation, infection, and sepsis: clinical utility and limitations. Crit Care Med 36:941-952, 2008

2. Berry MJA, Linoff GS: Data Mining Techniques: For Marketing, Sales, and Customer Support. New York: Wiley, 1997

3. Connolly ES Jr, Rabinstein AA, Carhuapoma JR, Derdeyn CP, Dion J, Higashida RT, et al: Guidelines for the management of aneurysmal subarachnoid hemorrhage: a guideline for healthcare professionals from the American Heart Association/American Stroke Association. Stroke 43:1711-1737, 2012

4. de Rooij NK, Greving JP, Rinkel GJ, Frijns CJ: Early prediction of delayed cerebral ischemia after subarachnoid hemorrhage: development and validation of a practical risk chart. Stroke 44:1288-1294, 2013

5. Fisher CM, Kistler JP, Davis JM: Relation of cerebral vasospasm to subarachnoid hemorrhage visualized by computerized tomographic scanning. Neurosurgery 6:1-9, 1980

6. Fries M, Kunz D, Gressner AM, Rossaint R, Kuhlen R: Procalcitonin serum levels after out-of-hospital cardiac arrest. Resuscitation 59:105-109, 2003

7. Grote E, Hassler W: The critical first minutes after subarachnoid hemorrhage. Neurosurgery 22:654-661, 1988

8. Hayashida H, Kaneko T, Kasaoka S, Oshima C, Miyauchi T, Fujita M, et al: Comparison of the predictability of neurological outcome by serum procalcitonin and glial fibrillary acidic protein in postcardiac-arrest patients. Neurocrit Care 12:252-257, 2010

9. Höllig A, Remmel D, Stoffel-Wagner B, Schubert GA, Coburn M, Clusmann H: Association of early inflammatory parameters after subarachnoid hemorrhage with functional outcome: a prospective cohort study. Clin Neurol Neurosurg 138:177-183, 2015

10. Hunt WE, Hess RM: Surgical risk as related to time of intervention in the repair of intracranial aneurysms. J Neurosurg 28:14-20, 1968

11. Jennett B, Teasdale G, Braakman R, Minderhoud J, KnillJones R: Predicting outcome in individual patients after severe head injury. Lancet 1:1031-1034, 1976

12. Juvela S, Kuhmonen J, Siironen J: C-reactive protein as predictor for poor outcome after aneurysmal subarachnoid haemorrhage. Acta Neurochir (Wien) 154:397-404, 2012

13. Kruyt ND, Biessels GJ, de Haan RJ, Vermeulen M, Rinkel GJ, Coert B, et al: Hyperglycemia and clinical outcome in aneurysmal subarachnoid hemorrhage: a meta-analysis. Stroke 40:e424-e430, 2009

14. Lee VH, Ouyang B, John S, Conners JJ, Garg R, Bleck TP, et al: Risk stratification for the in-hospital mortality in subarachnoid hemorrhage: the HAIR score. Neurocrit Care 21:14-19, 2014

15. McGirt MJ, Mavropoulos JC, McGirt LY, Alexander MJ, Friedman AH, Laskowitz DT, et al: Leukocytosis as an independent risk factor for cerebral vasospasm following aneurysmal subarachnoid hemorrhage. J Neurosurg 98:1222-1226, 2003

16. Muroi C, Hugelshofer M, Seule M, Tastan I, Fujioka M, Mishima K, et al: Correlation among systemic inflammatory parameter, occurrence of delayed neurological deficits, and outcome after aneurysmal subarachnoid hemorrhage. Neurosurgery 72:367-375, 2013

17. Muroi C, Seule M, Sikorski C, Dent W, Keller E: Systemic interleukin-6 levels reflect illness course and prognosis of patients with spontaneous nonaneurysmal subarachnoid hemorrhage. Acta Neurochir Suppl 115:77-80, 2013

18. O'Connor E, Venkatesh B, Mashongonyika C, Lipman J, Hall $\mathrm{J}$, Thomas P: Serum procalcitonin and C-reactive protein as markers of sepsis and outcome in patients with neurotrauma and subarachnoid haemorrhage. Anaesth Intensive Care 32:465-470, 2004 
19. Podgorelec V, Kokol P, Stiglic B, Rozman I: Decision trees: an overview and their use in medicine. J Med Syst 26:445463, 2002

20. Rincon F, Gordon E, Starke RM, Buitrago MM, Fernandez A, Schmidt JM, et al: Predictors of long-term shunt-dependent hydrocephalus after aneurysmal subarachnoid hemorrhage. Clinical article. J Neurosurg 113:774-780, 2010

21. Rivero Rodríguez D, Scherle Matamoros C, Fernández Cúe L, Miranda Hernández JL, Pernas Sánchez Y, Pérez Nellar $\mathrm{J}$ : Factors associated with poor outcome for aneurysmal subarachnoid haemorrhage in a series of 334 patients. Neurologia 32:15-21, 2017

22. Satomi J, Ghaibeh AA, Moriguchi H, Nagahiro S: Predictability of the future development of aggressive behavior of cranial dural arteriovenous fistulas based on decision tree analysis. J Neurosurg 123:86-90, 2015

23. Spallone A, Acqui M, Pastore FS, Guidetti B: Relationship between leukocytosis and ischemic complications following aneurysmal subarachnoid hemorrhage. Surg Neurol 27:253258, 1987

24. Srinivasan A, Aggarwal A, Gaudihalli S, Mohanty M, Dhandapani M, Singh H, et al: Impact of early leukocytosis and elevated high-sensitivity $\mathrm{C}$-reactive protein on delayed cerebral ischemia and neurologic outcome after subarachnoid hemorrhage. World Neurosurg 90:91-95, 2016

25. Teasdale GM, Drake CG, Hunt W, Kassell N, Sano K, Pertuiset B, et al: A universal subarachnoid hemorrhage scale: report of a committee of the World Federation of Neurosurgical Societies. J Neurol Neurosurg Psychiatry 51:1457, 1988

26. Vergouwen MD, Vermeulen M, van Gijn J, Rinkel GJ, Wijdicks EF, Muizelaar JP, et al: Definition of delayed cerebral ischemia after aneurysmal subarachnoid hemorrhage as an outcome event in clinical trials and observational studies: proposal of a multidisciplinary research group. Stroke 41:2391-2395, 2010

27. Wilson DA, Nakaji P, Abla AA, Uschold TD, Fusco DJ, Oppenlander ME, et al: A simple and quantitative method to predict symptomatic vasospasm after subarachnoid hemorrhage based on computed tomography: beyond the Fisher scale. Neurosurgery 71:869-875, 2012
28. Witsch J, Frey HP, Patel S, Park S, Lahiri S, Schmidt JM, et al: Prognostication of long-term outcomes after subarachnoid hemorrhage: the FRESH score. Ann Neurol 80:46-58, 2016

\section{Disclosures}

The Helmut Horten and the Gebert Ruef Foundations funded this study.

\section{Author Contributions}

Conception and design: Hostettler, Schmid, Germans, Keller. Acquisition of data: Hostettler, Muroi, Richter, Neidert, Boss, Pangalu, Keller. Analysis and interpretation of data: Hostettler, Neidert, Keller. Drafting the article: Hostettler, Richter, Neidert, Keller. Critically revising the article: Hostettler, Muroi, Richter, Neidert, Seule, Pangalu, Germans, Keller. Reviewed submitted version of manuscript: Hostettler, Muroi, Schmid, Neidert, Seule, Boss, Pangalu, Germans, Keller. Approved the final version of the manuscript on behalf of all authors: Hostettler. Statistical analysis: Hostettler, Schmid. Administrative/technical/material support: Keller. Study supervision: Keller.

\section{Supplemental Information Previous Presentations}

Portions of this work were presented as a talk at the Annual Conference on Intracranial Pressure, Brain Perfusion, and Hydrocephalus, German Society for Neurosurgery (DGNC), Bern, Switzerland, November 2016; and at the Annual Conference on Intensive Care and Neuro-Traumatology, German Society for Neurosurgery (DGNC), Mannheim, Germany, February 2017.

\section{Correspondence}

Isabel Hostettler: University College London, United Kingdom. isabel.hostettler@gmail.com. 